\title{
Short Video Marketing During the EPIDEMIC
}

\author{
Jiayi $\mathrm{Xu}^{1, \mathrm{a},{ }^{*}, \dagger}$, Jiayi Chen ${ }^{2, \mathrm{~b}, *}, \dagger$, Lukuan $\mathrm{Liu}^{3,}{ }^{3},{ }^{*}, \dagger$ \\ ${ }^{1}$ International class, Jinan Xinhang Experimental Foreign Language School, Jinan, China \\ ${ }^{2}$ University of Glasgow (Glasgow International College) ,UK \\ ${ }^{3}$ Ywies International Education, Guangzhou, China \\ *Corresponding author email: a3442467832@qq.com, b2770153652@qq.com,cl13223117505@163.com \\ †The first two authors contributed equally. The Third author contributed less.
}

\begin{abstract}
After the outbreak of the Covid-19 epidemic, China has implemented a stay-at-home policy, and the rise of short videos led to a large number of businesses which started using short videos for marketing. The popularity of short videos during the epidemic has made us pay more attention to its marketing strategies. Among them, the marketing strategy of short videos utilize a lot of professional knowledge, such as the area in internet celebrity economy, vanity effect, herd effect and hunger marketing. In addition, short video marketing also uses a lot of technological expertise, such as the calculation of big data and the visualization of text. Also people get spiritual happiness while shopping. This study is going to use some prior findings and find a new way of outlook into the marketing strategy of short videos during the epidemic, and its future aspirations.
\end{abstract}

Keywords: Tik-tok, short video marketing, Covid-19 epidemic, marketing strategies

\section{INTRODUCTION}

With the advancement of the internet, people's living habits have also changed. With the rise of online merchants, the trading volume of Taobao and Tmall during the Double Eleven Festival in 2019 reached 268.4 billion yuan. Especially after the outbreak of the epidemic, China implemented a home policy, people stay at home and want to obtain daily necessities which must rely on online shopping. The opportunity of online business is at hand, and other offline stores are not far behind in launching online businesses. The rise of Tiktok short video has given many manufacturers a new way out. The rapid development of the short video sales industry has resulted in many previously unknown industries. Tik-tok's e-commerce's annual merchandise turnover in 2020 exceeded 500 billion yuan, more than three times than that of 2019. By jumping to other platforms, the entire e-commerce industry has reached a new peak. Someone has counted that Li Jiaqi, a popular beauty blogger, can earn hundreds of millions of dollars in a live broadcast. Whether it is a novel form of delivery or a lower price, shoppers are all attracted[1]. This research found these phenomenons interesting and tries to investigate the marketing strategies behind it. The previous research will be classified by theme, which comprises short video marketing and psychology, short video marketing and technology, as well as the critical observations of the disadvantages of short video marketing, and some future outlooks are proposed.

\section{SHORT VIDEO MARKETING AND PSYCHOLOGY}

During the epidemic, China stipulates that it is not necessary to go out and implement a stay-at-home policy. Whether it is food, clothing, housing and transportation, it needs to be purchased online. Businesses have taken a fancy to the business opportunity of live streaming. People stay at home and have more free time. In order to pass the time, the time spent on video sites has risen sharply. Businesses have seized this business opportunity and launched a live broadcast, allowing people to realize the convenience of online shopping while increasing their turnover. In order to make network marketing more fruitful, businesses use a lot of psychological skills behind the scene.

\subsection{Internet celebrity economy}

An internet celebrity refers to a person who has become popular by internet citizens because of an event or behavior in reality or online or a person who continues to export professional knowledge and become popular for 
a long time. Their popularity is due to the fact that certain characteristics of themselves are magnified under the influence of the internet, and they are in line with the psychology of internet citizens' aesthetics, ugliness, entertainment, stimulation, voyeurism, imagination, taste, and spectators. They are deliberately or unintentionally sought after by the online world., and has become a "internet celebrity" [2]. Behind the network, celebrities are often boosted by the team, carefully planned, and they attract the attention of others. Internet celebrities can be divided into fashion celebrities who are born on comprehensive social platforms, and are mainly evolved from small models, designers or Taobao sellers. They are often young and beautiful, and they know how to wear fashionable clothes, showing good socializing skills, where they interact nicely with fans. The form of monetization is e-commerce monetization; content influencers are from short video/audio platforms, who are mostly represented by self-media, using Weibo, WeChat public account, video and audio product channels as carriers, and output original content articles, paragraphs, comments, comics, videos, etc. The monetization forms are mainly content monetization, payment for services, offline activities etc.; anchor internet celebrityprofessional anchor born on the live broadcast platform, through the display of talents, output knowledge content, real-time strong interaction with the video and voice of the fans, the main disguised way is to reward the fans, naming, etc[3]. With the development of the internet, the internet celebrity economy is developing rapidly during this period. People can find their favorite products just by scanning short videos without leaving their homes. Ecommerce companies capture people's psychology and make small videos beautifully and interestingly. For example, some small videos do not sell items at the beginning, but accumulate popularity in the form of stories, and then start selling items later. In addition, some internet celebrities and e-commerce companies have reached an agreement to promote products to the masses in live broadcasts. E-commerce companies grasp the consumption preferences and consumption needs of fans, and convert the attention of fans into purchasing power, so as to achieve an economic model of monetization of traffic or content. The internet celebrity economy has two seemingly opposite theories in psychology, one is called the Snob Effect and the other is called the Bandwagon Effect. People hope that the things they buy are unique, they are especially attracted by the limited products that are advertised on the internet, but at the same time they see that the inventory is low and the number of people who are snapping up is too large to buy.

\subsection{Hunger Marketing}

In many marketing strategies, the most common one is hunger marketing. Short video marketing captures people's psychology and uses hunger marketing to expand sales. Hunger marketing is often caused by suppliers deliberately lowering production or creating an atmosphere of scarcity. It is by creating a scene where demand exceeds supply. More and more businesses on the internet are beginning to use "hunger marketing" to create the illusion of low output and low demand, and then they make high profits by raising prices. Manufacturers of short video marketing are of no exception. Shopping cart link snapping is a good example. The anchors ask fans to swipe the number of likes, followers or gifts to a certain number, and then they will link the very low-priced products in the live broadcast room. Often these reduced-price products are limited, and people must rush to buy them if they want to get them. In order to obtain low-priced and scarce products, many people will enter the live broadcast room to like or brush gifts. But in the end, very few products are really available. Even some anchors will ask actors to pretend to get merchandise, but in fact there is no real merchandise to cheat likes and views.

\section{SHORT VIDEO MARKETING STRATEGY AND TECHNOLOGY}

The marketing of short videos cannot be lively or instantaneous. To do a good job in short video marketing, it is needed to establish a more scientific and rigorous system, with continuous content operation, and to stimulate users' social diffusion through better content creativity and to make smart Technology-driven marketing which can make better brand contents that meet the right people[4]. Short video websites and platforms have used many marketing strategies and techniques to help screen customers more effectively and increase the success rate of marketing.

\section{1 "Big Data Era"}

Big data is a large-scale data collection that greatly exceeds the capabilities of traditional database software tools in terms of acquisition, storage, management, and analysis. It has the four major categories of massive data scale, fast data circulation, diverse data types, and low value density. Features can accurately correspond to customer groups. In this era of the Internet, big data has become a method of rapid e-commerce marketing. The accurate big data pushes accurately record the data through the user's search history and preferences. It has become a great tool for merchants to sell. A new type of marketing method that uses the interactivity of digital information and network media to achieve marketing goals. The platform and the e-commerce company have reached an agreement that the e-commerce company will give the platform a certain fee, and the platform will promote their products for the e-commerce company. The platform selects the most likely purchasers through big data screening. When people stay on a web-page for a long time, the background data will be recorded. Big data analyzes and selects the types of this item, and then 
selects similar products from the products to be promoted. For example, when people usually see skin care products on Taobao, Tik-tok will push skin carerelated videos and advertisements for them.

\subsection{Marketing content}

Short video can attract people's attention, and there are especially some that are mostly attractive. In the context of the current internet, several types of short videos are very popular and have become the leaders in short video delivery. One is to share peoples' life in the form of a vlog, insert some advertising implants in the middle, and inadvertently plant grass products for the audience. The second is the funny category, which allows the audience to learn about the product while entertaining and relaxing. The third and most intuitive way is to evaluate[5]. Nowadays, many bloggers sell their products through evaluation. In Li Jiaqi's live broadcast room, he shows the audiences the color number of the lipstick and puts it on his mouth in the most intuitive way to show the audience his products. In addition to this intuitive method, novel marketing methods have always been sought. People will look at them because they are curious, which will increase the traffic, and the possibility of buying will increase.

\subsection{Short video visualization}

According to research data, the brain can process visual content more than 60,000 times faster than plain text. This is an analysis of human instinct from a physiological point of view, and I am more willing to accept short videos. At the same time, the current fast pace of life has given birth to the status quo of watching on the subway, watching in the toilet, and waiting for the train. Short and succinct short videos are more in line with the needs of the time-fragmented scenes of the current busy era. This means that brands using short videos as the language of communication with users will be easier to be accepted by the audience, and it will be easier to achieve the communication effect of the integration of quality and effectiveness[6]. The visualization of short videos makes people more impressed with advertising.

\section{DISADVANTAGES OF SHORT VIDEO MARKETING}

\subsection{Influence of cognitive preference}

Taking Tiktok as an example, on September 3, 2017, the data released by Tiktok for the first time showed that $85 \%$ of users are under 24 years old, and the main players and users are basically post-95s and post-00s. According to the survey, this group of people has not only the characteristics of decisive, rapid and responsive consumer psychology, but also the shortcomings of emotional impulse and conformity consumption. Online shopping is not like physical shopping. People shopping in physical stores can directly identify products. In a virtual network environment, people's perception of products is limited to pictures and videos, as well as other consumer reviews. A large number of inaccurate advertising videos in Tiktok will affect their consumption decisions. People have a herd mentality, and the cognitive preference of Tiktok short video shows this better. The cognitive preference of TikTok short videos is affected by the production and release of good content value, which will affect the viewing behavior of users, the effective interaction rate between users and the company's TikTok account, and the company's business monetization ability. To give a simple example, when people find a product on Tiktok, they don't care about it at first, but after they swipe it countless times and hear a lot of recommendations from bloggers, most of them will shake their minds, stimulate consumption, and have a desire to buy. However, when the buyers receive the goods and find that it does not meet their expectations, returns of goods and bad reviews will occur.

\subsection{Leading minors to blindly follow suit}

Because most of the audiences are young people, because young people are not deeply involved in the world, their psychology is not yet fully mature, and they are in a stage of being extremely vulnerable, so they are easy to be led by online content. Short videos are spreading fast, and misleading contents are also emerging one after another, such as performing vulgar, exaggerated content, spreading bad information, and touching the bottom line of the law, which are not conducive to the healthy growth of children. Even driven by strong curiosity, children will imitate the high-risk actions in the video, causing irreversible tragedies. Because the styles of short videos are varied, most of them rely on curious, stimulating and vulgar content to catch people's attention. In the case of imperfect mental and cognitive development of young people, it is difficult for them to make sound judgments on the quality of the content, with their blind faith and blind obedience, and values. The edges are gradually blurred. Many children have encountered pornographic information harassment in short videos, and they have been maliciously attacked, mocked, and insulted when they publish subjective remarks in the comment area. In the long run, these negative information will be regarded by teenagers as normal entertainment methods, resulting in direct influence on psychology.

\section{FUTURE OUTLOOK}

The degree of freedom of short videos will become increasingly higher. Businesses and platforms interact with videos in order to increase the constant curiosity of buyers, and will invisibly improve video advertisements to achieve the purpose of marketing. The number of 
downloads of short video software will increase rapidly, generating comments that interact with users in a short period of time, such as forwarding. Users can quickly create videos, and upload online video sites within a few minutes. Short video product information will become more and more creative, and novel advertising content and rich stories will appear.

\section{CONCLUSION}

The popularity of short videos during the epidemic has made us pay more attention to its marketing strategies. With the continuous upgrading of consumption, consumers are no longer satisfied with material consumption, but are more eager for emotional and spiritual satisfaction, hoping to obtain pleasure through consumption behavior. Interesting videos allow people to get spiritual happiness while shopping. At the same time, the realization of big data and short video production technology and marketing methods make it more valuable than the product itself. However, short video marketing also has its shortcomings. Therefore, the goal also needs to maximize the strengths and avoid the weaknesses, avoid problems, and match the virtual technology with reality. These is to ensure the positive nature of the content. Today, short videos have become a symbol of young people's personality and have important social significance. The short video marketing strategy points out the future development direction of marketing to a certain extent. Businesses should seize the spiritual consumption needs of consumers and formulate more targeted marketing strategies.

\section{References}

[1] Ma Yumei, Wu Yilin. Short video marketing in the era of mobile Internet [J]. China Statistics, 2018,No.444 12 28-30.

[2] Luo Xiaochun. Innovation and Reform of short video Marketing methods [J]. Science and Education Guide (Mid-day), 2018,No.335 11 180-181.

[3] Yongte, 2018-8, The past, present and future of the Internet celebrity economy, Zhihu , Https://zhuanlan.zhihu.com/p/50491248,

[4] 2019, "2019 Short Video Marketing White Paper", how to seize the marketing new wind mouth? , massive engine

Https://www.oceanengine.com/insight/2019duanshipin

[5] Cai Jie. Research on Short video Marketing Strategy in mobile Internet Era -- Taking a short video as an example [J]. West Leather, 2020,42(483), 18 100101.
[6] Ke Ji Cha Guan, 2017-11-15, the era of short video marketing has come, how should brand owners take this train? Everyone is a product experience, Http://www.woshipm.com/it/847656.html 